\title{
Hors des sentiers battus
}

\section{Philippe Lazar}

\section{ADRESSE}

Ph. Lazar : directeur général de l'Inserm. Institut national de la santé et de la recherche médicale, 101, rue de Tolbiac, 75654 Paris Cedex, France.

\section{TIRÉS A PART}

Ph. Lazar. a brillante réussite de médecine/sciences m'incite à utiliser la tribune qui m'est aimablement offerte par ses responsables à l'occasion de son cinquantième numéro pour donner un éclairage particulier du rôle original et pionnier de cette remarquable publication. Il s'agit, en effet, à mes yeux, de la première authentique revue d'enseignement supérieur et je voudrais montrer qu'elle pourrait, en cela, servir de modèle à beaucoup d'autres.

Après la dégradation progressive de l'effort national de recherche constatée au cours des années 1970, la décennie qui s'achève a été celle du redressement. Une politique ambitieuse, fondée sur trois volets complémentaires - un effort financier sans précédent, une consultation attentive des acteurs sociaux concernés, l'affirmation claire des exigences de la nation - a complètement modifié le tableau de notre potentiel de recherche et de développement. Il serait cependant contraire à la vérité de prétendre que tout va désormais pour le mieux dans le meilleur des mondes possibles et, s'il n'est pas dans mes intentions de dresser l'inventaire exhaustif des problèmes qui persistent, je voudrais néanmoins en évoquer deux : la situation précaire du français en tant que langue scientifique et la trop grande disparité de statut social des fonctions de recherche et d'enseignement supérieur. L'apport de médecine/sciences n'en apparaîtra que plus clairement, je le pense.

\section{L'anglophonie : mode ou nécessité ?}

Personne n'a jamais remporté une victoire en pratiquant la politique de l'autruche. Refuser de regarder en face les problèmes linguistiques de la communication scientifique n'a pas pour vertu secrète de les faire disparaître. La langue privilégiée des échanges directs - écrits ou oraux - entre scientifiques est, aujourd'hui, qu'on s'en réjouisse ou qu'on le déplore, cet anglo-américain abâtardi dans lequel nombre de sujets de sa Gracieuse Majesté refusent de reconnaître leur langue maternelle. Qui veut communiquer avec ses pairs du monde entier ne peut ignorer cette situation de fait, sauf à miser sur un protectionnisme des frontières, ce qui, en sciences plus encore peut-être que dans d'autres domaines, semble être un pari bien téméraire: la science est par essence universelle et impose, pour une large part, ses règles propres de développement à qui veut être et rester dans la course internationale au savoir et à ses applications. Cependant le constat qui vient d'être 


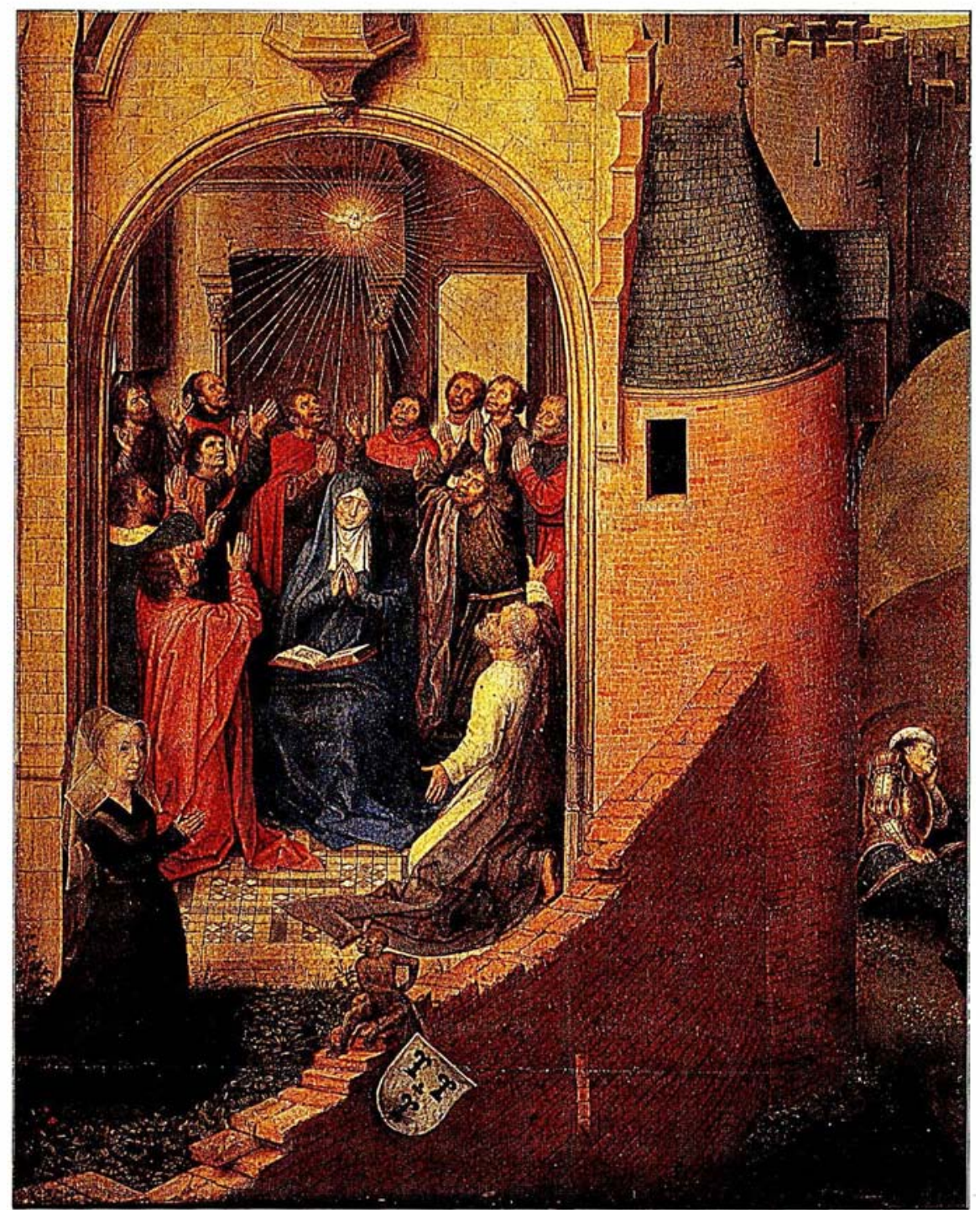

Pentecôte. Memling (1433-1494). Par la grâce du Saint-Esprit, les apôtres comprirent aisément toutes les langues. (Memling, La Pentecôte, Scala).

évoqué est descriptif et d'ordre général. Il ne dispense pas d'une analyse plus fine des raisons d'être de la situation actuelle et, par là-même, des bases imaginables de son évolution.

Le principal argument qui milite en faveur d'une langue unique de communication scientifique - et unicité signifie, de facto, anglophonie ! - est l'exigence de qualité de la recherche effectuée. Or il est bien clair que, sans résoudre toutes les difficultés, la 204 uñ terrain de dure joute internationale - au moyen d'une langue qui leur est nécessairement moins familière qu'à ceux qui l'ont absorbée dès leur naissance - serait avant tout le témoignage de leur désir effréné de prestige, voire même de leur snobisme (sic!). Si la volonté de voir leurs mérites reconnus fait incontestablement partie des motivations des chercheurs - mais peut-on leur en faire reproche alors même qu'on exalte à tous moments l'esprit de compétition ? - il serait absurde de ne pas comprendre la nécessité vitale pour eux d'établir des liens directs avec leurs pairs au travers du monde. Faute de le faire, ils prendraient le risque majeur de s'isoler très rapidement sur des voies inévitablement moins riches d'espérances de succès puisque ne bénéficiant pas d'échanges continus d'informations $\mathrm{au}$ sein des collèges invisibles que constituent les petites communautés internationales travaillant sur des sujets voisins.

L'argumentation qui précède est, aux yeux de beaucoup de scientifiques, si forte, qu'elle règle pour eux le problème. Soyons-en certes désolés, pensent-ils, mais acceptons la loi du genre : parlons et écrivons la science en anglais; tout le reste n'est que, sans doute, sympathique mais non moins irresponsable nostalgie...

\section{Une situation moins figée qu'on ne le pense}

Pris en bloc, ce plaidoyer " réaliste " en faveur de l'utilisation de l'anglais est difficile à contourner. Mais tout le problème n'est-il pas, précisément, de refuser, ici comme ailleurs, le monolithisme? En fait, la situation est plus nuancée qu'il ne peut paraître de prime abord.

En premier lieu, il existe d'incontestables différences entre les divers secteurs de la science, en fonction de leur histoire et de leur contenu. Il est clair que le tableau n'est pas le même en mathématiques ou en biologie, en sociologie ou en médecine. En second lieu, nous avons affaire aujourd'hui à des pratiques dont rien ne permet d'affirmer qu'elles sont définitives. L'évolution politique, pour le moins inattendue dans son intensité et sa vitesse, de l'Europe 


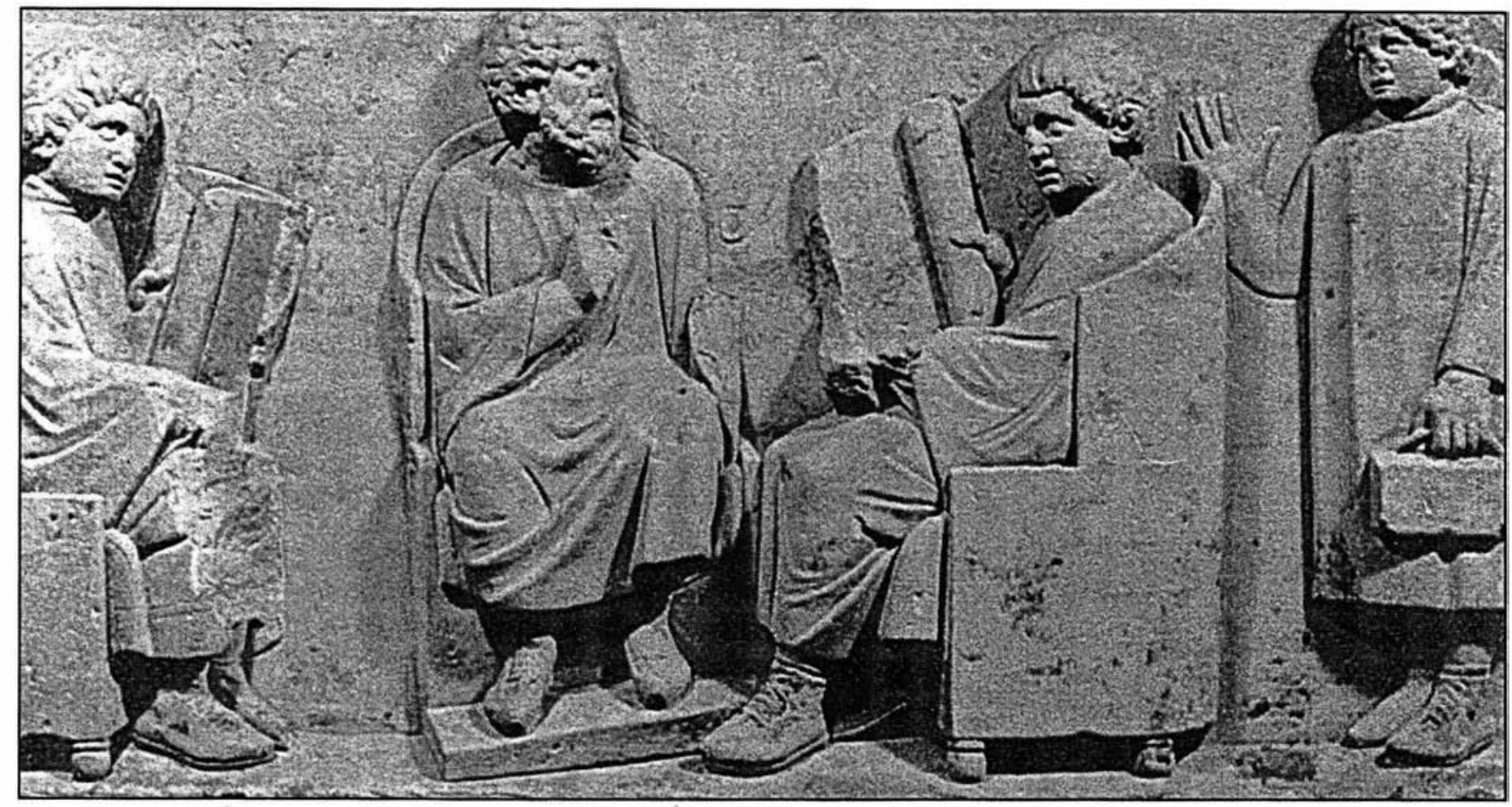

Le maître et ses élèves. (Musée de Trèves).

centrale au cours des tout derniers mois devrait renforcer notre conviction quant à la labilité potentielle des situations qui paraissent les plus solidement établies! Or deux arguments plaident ici en faveur de possibles changements. Dans un intéressant article publié le 16 janvier dernier, le journal Libération attirait l'attention sur un nouveau mouvement de fuite des cerveaux, qui s'amorce cette fois depuis les États-Unis vers plusieurs pays de l'Asie du Sud-Est, en liaison avec la puissance économique montante de ces derniers. Est-il absolument établi - n'en déplaise à Olivier Postel-Vinay qui stigmatisait récemment, non sans humour, nos efforts en faveur de la francophonie en les transposant en une imaginaire " nippophonie " (les Échos, 17 janvier 1990) - que les Japonais, précisément, accepteront pour les siècles des siècles de publier leurs résultats scientifiques les plus aigus en anglais ? Qu'on me permette au moins de poser la question... L'autre argu$\mathrm{m} / \mathrm{s} n^{\circ} 3$ vol. 6 , mars 90 ment, plus solide encore, en faveur d'une modification au long cours des pratiques actuelles, tient à la croissance explosive, de nos jours, de l'ensemble des informations scientifiques pertinentes, et à l'impérieuse nécessité de recourir systématiquement, dès un proche avenir, à une gestion informatisée de leur recueil et de leur diffusion si nous voulons éviter d'être complètement débordés. Or il est probable que, compte tenu des modalités spécifiques d'acquisition et de traitement des messages télématiques, susceptibles de supprimer à la source toute ambiguïté sur leur contenu et de permettre, de ce fait, des traductions automatiques systématisées, nous allons, sans doute plus vite que nous ne le pensons habituellement, vers un véritable bouleversement de nos pratiques informatives. Les préoccupations se déplaceront aussi sans doute pour partie de l'émission - aujourd'hui seule à être vraiment prise en compte - vers la réception des messages pertinents, et ce déplacement d'équilibre ne pourra s'effectuer indépendamment de l'usage des langues. Or nous avons encore, pour le moment, toutes nos chances en la matière, compte tenu notamment de l'excellence de l'école française d'informatique théorique. $\mathrm{Ne}$ devrions-nous pas accentuer délibérément nos efforts dans ce domaine?

\section{La restructuration du savoir, aujourd'hui et demain}

L'expression et la diffusion des connaissances nouvelles - le fruit de ce qu'on appelle, à proprement parler, la "recherche" - ne constituent cependant qu'une fraction minoritaire de l'information scientifique et technique. La faveur dont jouit aujourd'hui - en termes de valeurs reconnues - la recherche prise en ce sens réducteur tend malheureusement à occulter l'importance stratégique fondamentale d'une autre forme 
d'activité réflexive qui pourtant, compte tenu de l'évolution explosive déjà évoquée de la science, actuellement, devrait retenir en priorité notre attention et mobiliser nos efforts. Je veux parler de l'appréhension critique, du rassemblement, de la restructuration permanente, de la synthèse et de la transmission du savoir.

Chacun sait que l'honnête homme qui sait de tout un peu n'existe plus et qu'a fortiori l'" honnête savant" qui aurait intellectuellement accès à l'ensemble des branches du savoir, ne serait-ce qu'au sein de l'un quelconque des grands secteurs de la pensée, est désormais un être mythique. Il faut accepter, bon gré mal gré, une forte parcellisation de la connaissance.

Mais il n'en faut pas moins, pour autant, lutter contre le risque de fragmentation excessive dont nous sommes menacés du seul fait de l'hyperspécialisation des tâches, notamment dans le champ de la science. Tâche ardue, qui réclame compétence, ouverture d'esprit, capacité d'abnégation vis-à-vis de choix trop personnels de centres d'intérêt, esprit de synthèse ; tâche essentielle dès lors que l'on veut à la fois informer, faire comprendre et faire réfléchir de façon prospective aux conséquences à tirer de l'état présent des connaissances.

Qui n'aura reconnu, dans la description qui précède, la philosophie fondatrice d'un véritable enseignement supérieur? C'est bien à un exercice de cette nature que se livrent les universitaires qui veulent pleinement assumer ce qu'on appelle habituellement les charges et que je préfere appeler les spécificités de leur métier. C'est une tâche à l'évidence difficile, exaltante, et qui serait gratifiante aussi si l'on voulait bien la reconnaître comme l'une des formes privilégiées d'expression de la pensée scientifique et critique.

Or il est clair que tel n'est pas encore vraiment le cas dans notre pays, qui survalorise la recherche " analytique" par rapport à ce type d'activité réflexive et novatrice. C'est bien dommage, car on pourrait sans doute trouver là l'une des meilleures voies de revalorisation de la fonction d'évaluation des compétences des hommes et des équipes concernées. Pourquoi, en particulier, ne pas privilégier la prise en compte d'articles publiés dans des revues comme médecine/sciences - car on aura bien sûr reconnu, au travers de ma description théorique, la valeur paradigmatique de cette revue - pour établir les jugements de valeur dont ne peut se passer, de nos jours, une université désireuse de jouer pleinement son rôle ? Tout universitaire qui le souhaite doit évidemment pouvoir faire de la recherche analytique - et sa production scientifique doit naturellement être prise en compte dans sa carrière - mais tout universitaire devrait, en règle générale, contribuer à la restructuration permanente du savoir et voir ses efforts de publication dans des revues de synthèse reconnus et portés activement à son crédit. Il faudrait pour cela cesser d'affirmer que seule la fraction de l'activité des universitaires qui concerne leur participation à l'acquisition de connaissances analytiques nouvelles peut être

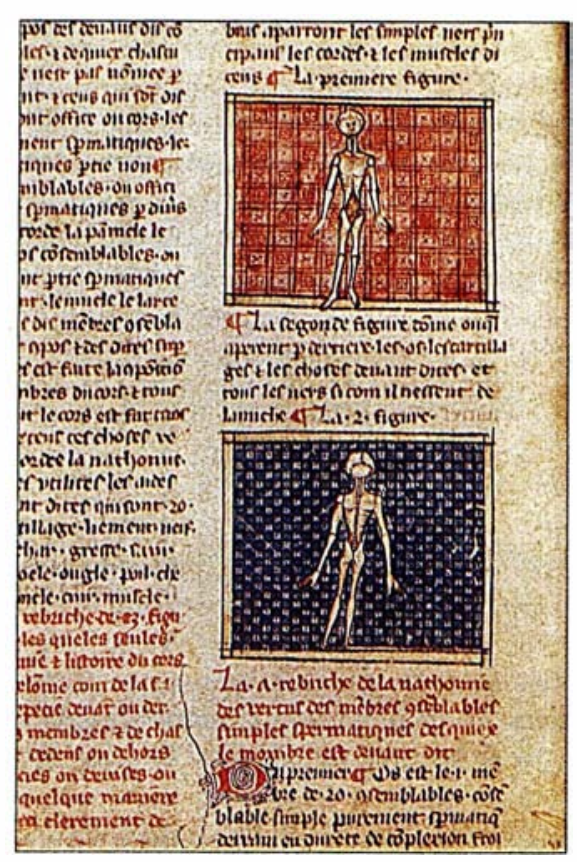

Manuscrit francais de 1314 de Henri de Mondeville. (voir légende de la page 193). (Ms Fr. 2030, chirurgien de Mondeville, Bibliothèque Nationale de Paris). mesurée et intervenir dans les jugements établis sur eux. L'existence, le niveau de qualité et le succès de médecine/sciences montrent clairement qu'il existe une voie alternative dans laquelle il serait urgent que nous nous engagions.

\section{Le francais, langue d'avenir}

Est-il besoin d'ajouter, à ce stade du raisonnement, à quel point une telle attitude contribuerait à la défense de la langue française en tant que langue scientifique? N'est-il pas évident, en effet, que des revues de synthèse et de restructuration du savoir, comme médecine/sciences, ne peuvent, par essence, être rédigées que dans la langue maternelle de leurs principaux lecteurs?

Au-delà de ce constat de simple bon sens, je voudrais ajouter que la tradition intellectuelle française - rationalité, cartésianisme - et son expression linguistique spécifique sont particulièrement bien adaptées à des activités de cette nature. Il est donc clair qu'une telle politique conférerait aux pays francophones des atouts supplémentaires de grande valeur pour affronter l'avenir: n'est-ce pas en misant sur les jeunes que, dans une période de bouleversement incessant du savoir, on se met de la façon la plus efficace en situation d'adaptation résolue au changement?

médecine/sciences montre qu'une telle politique ne relève pas d'une pure utopie, qu'elle pourrait même devenir le fondement d'un enseignement supérieur renouvelé, évoluant en synergie avec la recherche sans renoncer pour autant à sa mission première qui est de transformer, en quelques années, des bacheliers en " producteurs" de tous ordres, capables d'évoluer en fonction des transformations qui renouvelleront en permanence le monde dans lequel ils vivront et dont rien ne permet de penser que le rythme se ralentira au cours des décennies à venir. Que cette revue et ses responsables soient donc félicités et remerciés des perspectives stimulantes qu'ils offrent ainsi à notre réflexion et chaleureusement encouragés à poursuivre une œuvre, au sens fort du terme, exemplaire 